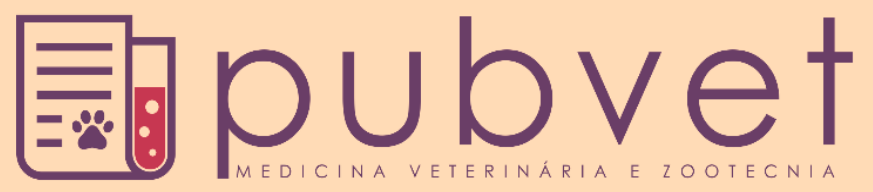

ISSN 1982-1263

https://doi.org/10.31533/pubvet.v15n03a770.1-7

\title{
A importância do diagnóstico precoce na doença do disco intervertebral (Hasen Tipo I) em canino: Relato de caso
}

\author{
Suélen Dalegrave ${ }^{1 *} \theta$, Matheus Marçal dos $\operatorname{Passos}^{2}{ }^{\circ}$, Laís Rezzadori Flecke ${ }^{30}$, Thaisa \\ Schroeder Dutra $^{4}$ ๑

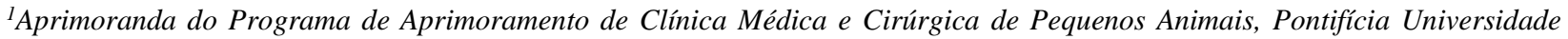 \\ Católica do Paraná, Toledo, Paraná, Brasil. \\ ${ }^{2}$ Médico veterinário, Neurologista, proprietário da clínica veterinária Ilha Animal, Rio de Janeiro, RJ, Brasil. \\ ${ }^{3}$ Aprimoranda do Programa de Aprimoramento de Clínica Médica de Pequenos Animais, Universidade de Caxias do Sul, Caxias \\ do Sul, Rio Grande do Sul, Brasil. \\ ${ }^{4}$ Médica veterinária, Anestesista da clínica veterinária Ilha Animal, Rio de Janeiro, RJ, Brasil. \\ *Autor para correspondência: suhdalegrave@hotmail.com
}

Resumo. A doença de disco intervertebral (DDIV) Hasen tipo I, ocorre pela extrusão do material do núcleo pulposo para o canal vertebral. A matriz do núcleo se degenera, desidrata e mineraliza, associando a degeneração condroide do disco intervertebral. Embora não se saiba a etiologia dessa alteração, acredita-se que a genética tenha grande importância. A DDIV pode desenvolver-se em qualquer segmento medular, porém a região toracolombar é mais acometida. A coluna vertebral torácica apresenta menor risco de herniação de disco, devido a estabilização por meio de ligamentos intercapitais dorsais. Acomete animais de meia idade a idosos e não tem predileção sexual. A doença é mais observada em raças condrodistróficas, mas também ocorre em raças de grande porte. O objetivo foi relatar o atendimento de um cão diagnosticado por tomografia com doença do disco intervertebral Hasen tipo I toracolombar em um canino. $\mathrm{O}$ animal chegou para atendimento neurológico encaminhado, apresentando histórico de claudicação do membro posterior esquerdo há 4 semanas. Paciente apresentando retenção urinária. Observou-se durante a avaliação clínica paraparesia ambulatorial e déficit proprioceptivo nos testes de postura e saltitamento de membro posterior esquerdo. Dor superficial e profunda preservadas. Reflexos segmentares espinhais em membros pélvicos apresentando hiperreflexia patelar esquerda. Tônus muscular sem alterações no momento do exame. Encaminhado para tomografia computadorizada no qual constatou compressão por meio de extrusão em T12-T13 ventrolateral esquerda, submetido a cirurgia com melhora do quadro. Este trabalho demonstra a importância do diagnóstico precoce, por meio de um neurológico correto, exames de imagem e assim uma conduta terapêutica cirúrgica eficaz, com resultado da melhora do quadro clínico do paciente.

Palavras-chave: Cão, diagnóstico, doença do disco intervertebral, neurologia veterinária

\section{The importance of early diagnosis in canine thoracolumbar intervertebral disc disease (Hasen Type I): Case report}

Abstract. Hasen type I intervertebral disc disease (DDIV) occurs due to the extrusion of material from the pulpal nucleus into the vertebral canal. The matrix of the nucleus degenerates, dehydrates and mineralizes, associating chondroid degeneration of the intervertebral disc. 
Although the etiology of this alteration is not known, genetics is believed to be of great importance. DDIV can develop in any spinal segment, but the thoracolumbar region is more affected. The thoracic spine has a lower risk of disc herniation, due to stabilization through dorsal intercapital ligaments. It affects middle-aged animals to the elderly and has no sexual predilection. The disease is most commonly seen in chondrodystrophic breeds, but it also occurs in large breeds. The objective was to report the care of a dog diagnosed by tomography with Hasen type I thoracolumbar intervertebral disc disease in a canine. The animal arrived for referred neurological care, presenting a history of claudication of the left posterior limb for 4 weeks. Patient showing urinary retention. During the clinical evaluation, outpatient paraparesis and proprioceptive deficit were observed in the tests of posture and jumping of the left posterior limb. Superficial and deep pain preserved. Spinal segmental reflexes in pelvic limbs showing left patellar hyperreflexia. Muscle tone without changes at the time of the exam. He was referred for computed tomography in which he found compression by means of extrusion on the left ventrolateral T12-T13, who underwent surgery with improvement of the condition. This work demonstrates the importance of early diagnosis, by means of a correct neurological, imaging exams and thus an effective surgical therapeutic approach, with the result of improving the patient's clinical condition.

Keywords: Dog, diagnosis, intervertebral disc disease, veterinary neurology

\title{
La importancia del diagnóstico precoz en la enfermedad del disco intervertebral toracolumbar (Hasen Tipo I) en canino: Reporte de caso
}

\begin{abstract}
Resumen. La enfermedad del disco intervertebral (DDIV) Hasen tipo I se produce debido a la extrusión de material del núcleo pulpar hacia el canal vertebral. La matriz central degenera, deshidrata y mineraliza, asociando la degeneración condroide del disco intervertebral. Aunque se desconoce la etiología de esta alteración, se cree que la genética es de gran importancia. El DDIV puede desarrollarse en cualquier segmento espinal, pero la región toracolumbar está más afectada. La columna torácica tiene un menor riesgo de hernia discal, debido a la estabilización a través de los ligamentos intercapitales dorsales. Afecta a animales de mediana edad a ancianos y no tiene predilección sexual. La enfermedad se observa con mayor frecuencia en razas condrodistróficas, pero también ocurre en razas grandes. El objetivo fue reportar el cuidado de un perro diagnosticado por tomografía con enfermedad del disco intervertebral toracolumbar tipo I de Hasen en un canino. El animal llegó para atención neurológica referida, presentando un antecedente de claudicación de la extremidad posterior izquierda durante 4 semanas. Paciente que presento retención urinaria. Durante la evaluación clínica se observó paraparesia ambulatoria y déficit propioceptivo en las pruebas de postura y salto del miembro posterior izquierdo. Dolor superficial y profundo preservado. Reflejos espinales segmentarios en extremidades pélvicas mostrando hiperreflexia rotuliana izquierda. Tono muscular sin cambios en el momento del examen. Fue remitido para tomografía computarizada en la que encontró compresión mediante extrusión en el ventrolateral izquierdo T12-T13, quien fue intervenido quirúrgicamente con mejoría del cuadro. Este trabajo demuestra la importancia del diagnóstico precoz, mediante un correcto examen neurológico, de imagen y por tanto un abordaje terapéutico quirúrgico eficaz, con el resultado de mejorar la situación clínica del paciente.
\end{abstract}

Palabras clave: Perro, diagnóstico, enfermedad del disco intervertebral, neurología veterinaria

\section{Introdução}

A doença do disco intervertebral (DDIV) é uma condição comum que pode afetar a coluna vertebral de cães adultos e idosos (Dewey \& Costa, 2016). A compreensão da medula espinhal se dá, por consequência, 
do deslocamento do disco intervertebral para o canal vertebral, podendo ocorrer na forma de extrusão Hansen tipo I, protrusão - Hansen tipo II (Bergknut et al., 2013; Hansen, 1951, 1952), extrusão de núcleo pulposo não compressivo agudo (Risio et al., 2009), extrusão do núcleo pulposo hidratado (Falzone, 2017) e extrusão do disco intervertebral intradural/intramedular (Fenn et al., 2020; Risio, 2015).

A herniação de disco tipo I ocorre pela extrusão do material do núcleo pulposo para o canal vertebral. A matriz do núcleo se degenera, desidrata e mineraliza, associando a degeneração condroide do disco intervertebral (Ettinger et al., 2002; N D Jeffery et al., 2013; Taylor, 2015). Embora não se saiba a etiologia dessa alteração, acredita-se que a genética tenha grande importância (Ettinger et al., 2002). As opções terapêuticas incluem o tratamento médico e cirúrgico (Moore et al., 2020). No tratamento médico, o material do disco extruído é reabsorvido pelo processo inflamatório, pela ativação dos macrófagos e ocorre a cicatrização do ânulo fibroso rompido (Dewey \& Costa, 2016).

A intervenção cirúrgica é indicada quando não há resposta ao tratamento conservador, quando os sinais clínicos são recidivantes ou progressivos e em caso de paresia não ambulatória e paraplegia com ou sem nocicepção (Brisson, 2010; Moore et al., 2020).

O presente estudo objetivou relatar a eficiência de um diagnóstico precoce e assertivo para a DDIV e assim ter um tratamento de sucesso com um prognóstico favorável para o paciente.

\section{Relato de caso}

Um canino, sem raça definida, macho, castrado, com 5,5 $\mathrm{kg}$ e cinco anos de idade chegou para atendimento com histórico de claudicação do membro posterior esquerdo há quatro semanas e retenção urinária há um dia. Foi atendido por outro serviço veterinário, há duas semanas, que prescreveu repouso absoluto, prednisolona $1 \mathrm{mg} / \mathrm{kg}$, à cada 12 horas, omeprazol $0,5 \mathrm{mg} / \mathrm{kg}$, à cada 24 horas e gabapentina 10 $\mathrm{mg} / \mathrm{kg}$, à cada 12 horas; não apresentando melhora clínica.

Ao exame físico, o paciente demonstrava-se alerta, sem alterações em parâmetros fisiológicos. No exame neurológico constatou-se, que animal se apresentava sem alterações comportamentais e em pares de nervos cranianos; paraparesia ambulatorial (Grau II), reflexos segmentares espinhais em membros pélvicos (patelar, flexor e perineal) apresentando apenas hiper-reflexia patelar esquerda, demais reflexos normais, reações posturais avaliadas através de testes de propriocepção e teste do salto com déficit em membro pélvico esquerdo. Apresentava tônus muscular em normal, dor a palpação epaxial entre os segmentos T3L3 e presença de dor profunda nos membros pélvicos.

Como exames complementares foram solicitados exames hematológicos, o cateterismo vesical com posterior urinálise, tendo como resultado dentro dos padrões para espécie. A radiografia simples da coluna vertebral toracolombar foi indicada, mas tutores se negaram à realização.

Na tomografia computadorizada, revelou compressão extramedular ventrolateral entre T12-T13, lado esquerdo ocupando cerca de $80 \%$ do canal vertebral (Figuras 1A e 1B). Outro achado interessante foi, a presença de arco costal flutuante à direita e hipoplásico a esquerda em L1 (Figura 1C).

Frente ao histórico, achados clínicos, neurológicos e exames complementares, o diagnóstico foi de doença do disco intervertebral, optando-se pelo encaminhamento cirúrgico do paciente para descompressão da medula espinhal por meio da técnica de hemilaminectomia.

Depois de anestesiado, o animal foi posicionado em decúbito esternal com membros torácicos e pélvicos flexionados, foi realizada a antissepsia do campo operatório com posterior incisão de pele, subcutâneo e musculatura epaxial dorso-lateral esquerda, na localização das extensões vertebrais entre T12 e T13. Procedeu-se ao afastamento da musculatura local para visualização da lâmina vertebral entre T12-T13, lado esquerdo. Com auxílio de uma goiva, removeu-se o processo articular. O desgaste da lâmina lateral ocorreu com Drill pneumático, concomitante a instilação de solução fisiológica. Após o desgaste da parte cortical interna, foi realizada sua remoção com auxílio de uma pinça de Kerrinson. Posterior a remoção do conteúdo extruído do disco (Figura 2), foi realizada a fenestração do disco intervertebral entre T12-T13. A síntese da 
musculatura ocorreu com polidiaxonona 3-0 em padrão contínuo simples. A sutura de subcutâneo ocorreu em padrão "Walking Suture", com o mesmo fio e a dermorrafia foi obtida em padrão Sultan utilizando-se Nylon 4-0.
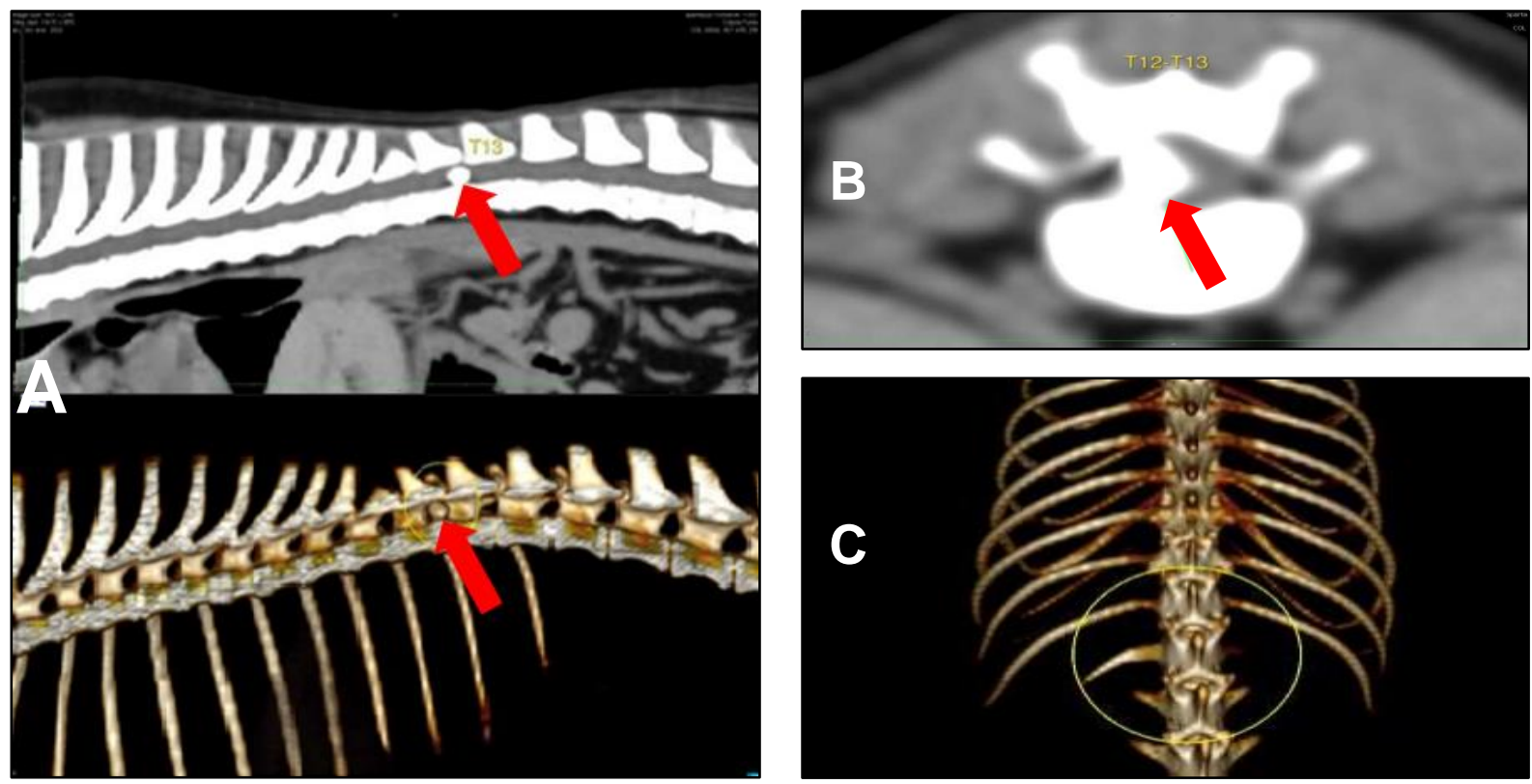

Figura 1. Tomografia de coluna vertebral de canino, apresentando compressão medular entre as vértebras T12 e T13 ventrolateral esquerda indicado pela flecha (A); região ventrolateral esquerda (flecha), entre as vértebras T12 e T13 (B) e Tomografia de coluna vertebral de canino, apresentando arco costal flutuante à direita e hipoplásico à esquerda, em L1 (C).

Na prescrição pós-operatória instituiu-se o uso de metadona $0,3 \mathrm{mg} / \mathrm{kg}$, quatro vezes ao dia, dipirona 25 $\mathrm{mg} / \mathrm{kg}$, intravenoso, três vezes ao dia e dexametasona $0,3 \mathrm{mg} / \mathrm{kg}$, intravenoso, dose única. O paciente teve alta hospitalar após dois dias, devido a boa recuperação anestésica e ausência de intercorrências trans e pósoperatórias. Para prescrição de alta, utilizou-se tramadol $5 \mathrm{mg} / \mathrm{kg}$, via oral, quatro vezes ao dia, durante cinco dias e dipirona $25 \mathrm{mg} / \mathrm{kg}$, três vezes ao dia; paciente já havia urinado sozinho antes da alta hospitalar. No retorno, após 15 dias, pode-se observar uma melhora na locomoção e sinais clínicos e neurológicos do paciente, apenas apresentando uma discreta ataxia no membro pélvico esquerdo.

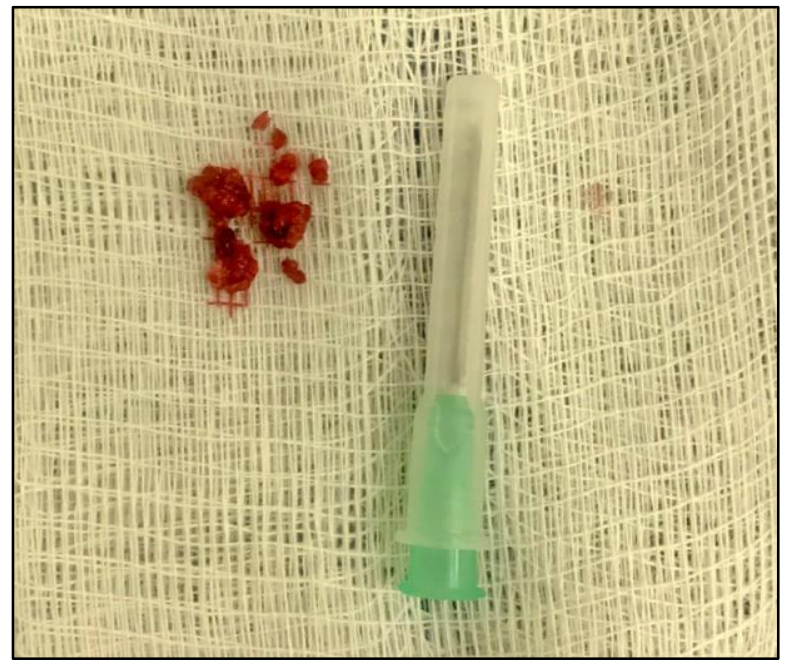

Figura 2. Material extruído do núcleo pulposo, retirado cirurgicamente do canal vertebral. 


\section{Discussão}

A doença do disco intervertebral pode ocorrer devido a uma degeneração de disco, que acomete geralmente animais entre três à sete anos de idade e raças condrodistróficas (Aikawa et al., 2012; Aikawa, et al., 2012; Dewey \& Costa, 2016). Embora, Brisson (2010) indicou que a incidência entre gêneros seja semelhante, alguns autores relataram a predisposição em machos (Aikawa et al., 2012). O paciente do relato apresentava idade e gênero compatível com a prevalência observada pela maioria dos autores, entretanto tratava-se de um animal sem raça definida.

Autores relataram maior incidência da afecção em cães em região toracolombar (46,5\%), pelo fato do canal vertebral ser quase inteiramente preenchido pela medula espinhal nessa região (Innes \& Melrose, 2015). A compressão extramedular é comumente visualizada entre os espaços intervertebrais T11 e L3 (Dewey \& Costa, 2016). O paciente, apresentava compressões entre T12-T13 o que corrobora com o estudo em questão.

A doença do disco intervertebral, com base na severidade da disfunção neurológica, pode ser classificada em graus que variam de um a cinco, sendo: Grau I - somente dor à palpação epaxial; Grau II - paraparesia ambulatória; Grau III - paraparesia não ambulatória; Grau IV - paraplegia com presença de dor profunda; Grau V - paraplegia com ausência de nocicepção. O paciente foi classificado quanto ao grau de disfunção neurológica em Grau II, uma vez que apresentava ataxia e paraparesia ambulatória (Martin et al., 2020; Sharp \& Wheeler, 2005). Os distúrbios na micção, poderiam estar associadas a localização toracolombar de neurônio motor superior. A bexiga espástica e difícil de esvaziar ocorre, devido ao aumento do tônus do esfíncter e a anulação do reflexo detrusor (Dewey \& Costa, 2016). A cateterização vesical auxilia na diminuição da ocorrência de infecção do trato urinário (Olby et al., 2010) a qual, foi realizada no paciente relatado, não apresentando sinais de infecção.

O diagnóstico presuntivo de DDIV toracolombar Hansen tipo I, baseia-se na resenha, na anamnese e no exame físico e neurológico, no qual foi realizado no paciente. Tais métodos sugerem a presença de DDIV, entretanto não descartam a presença de outras doenças como condições inflamatórias, infecciosas e neoplásicas (Costa et al., 2020). O diagnóstico definitivo baseia-se, em exames complementares como: mielografia, mielotomografia, tomografia computadorizada (TC), e ressonância magnética (RM) (Costa et al., 2020; Moore et al., 2020).

A TC escolhida como método diagnóstico nesse caso, permitiu a observação de mineralização de discos, e pode ser realizada de forma não invasiva, diferentemente do exame histopatológico, o exame também possibilitou saber a localização exata do material, que no caso era ventrolateral esquerda e a presença de arco costal flutuante direito e hipoplásico esquerdo auxiliando assim, para posterior acesso cirúrgico (Costa et al., 2020).

O tratamento clínico instituído inicialmente não obteve resultados satisfatórios. Em virtude disso, o animal foi encaminhado para tratamento cirúrgico como objetivo de remover o material que estava comprimindo a medula espinhal corroborando com os autores Sharp \& Wheeler (2005) e Brisson (2010). As principais técnicas cirúrgicas empregadas na coluna toracolomabar incluem: laminectomia dorsal (Hankin et al., 2012), hemilaminectomia (Moore et al., 2020) e corpectomia lateral (Salger et al., 2014). Como modo de prevenção de recidivas, indica-se a fenestração do disco intervertebral (Innes \& Melrose,

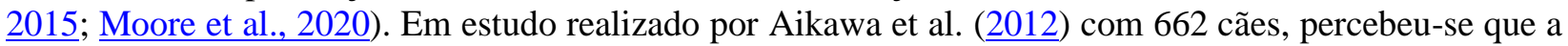
prevalência na recidiva de DDIV em discos não fenestrados, foi 26 vezes maior que nos discos fenestrados. O objetivo da técnica de fenestração, é remover todo o núcleo pulposo remanescente com potencial de herniar dorsalmente através do anel rompido após a cirurgia descompressiva (Aikawa, et al., 2012; Moore et al., 2020), optou-se pela realização da técnica no paciente em questão.

Em um estudo realizado por Martin et al. (2020) com 273 casos, foram separado dois grupos onde um deles sendo operado no mesmo dia da consulta e outro grupo não operou, aguardando para o dia posterior, como resultado os que foram operados na sequência (mesmo dia) apenas $4,5 \%$ perderam dor profunda e os demais que aguardaram para a cirurgia $12 \%$ perderam a dor profunda e a maioria não voltou a caminhar. 
Em outro relato de Castel et al. (2017) animais que foram operados após 12 horas, aumentaram a chance de mielomalácia. Com isso, observamos a importância de um diagnóstico precoce e assertivo para a realização o mais rápido possível da descompressão cirúrgica.

O prognóstico depende de diversos fatores como: a técnica cirúrgica empregada, o tempo de perda de nocicepção e a velocidade da perda dos movimentos voluntários. Pacientes que mantêm a percepção de dor profunda após a submissão cirúrgica, apresentam prognóstico bom a excelente, enquanto os que apresentarem perda de nocicepção, prognóstico ruim (Dewey \& Costa, 2016; Jeffery et al., 2016; Taylor, 2015). No presente relato, o paciente se mostrou com prognóstico favorável, visto que não apresentou perda da nocicepção e após a cirurgia obteve melhora em seu quadro urinário, locomoção e reações posturais.

\section{Conclusão}

O conhecimento da doença do disco intervertebral é de suma importância, pois a sua incidência na medicina veterinária é alta. Sua etiologia, sinais clínicos, diagnóstico, tratamento e prognóstico continuam sendo um desafio para o clínico geral.

A avaliação do paciente, por especialistas em neurologia, se demonstrou de suma importância para um diagnóstico assertivo e precoce, com posterior tratamento cirúrgico e prognóstico favorável. A colaboração dos tutores frente ao tratamento instituído e agilidade na busca por atendimento veterinário foram também, fundamentais para o sucesso terapêutico.

\section{Referências}

Aikawa, T., Fujita, H., Kanazono, S., Shibata, M., \& Yoshigae, Y. (2012). Long-term neurologic outcome of hemilaminectomy and disk fenestration for treatment of dogs with thoracolumbar intervertebral disk herniation: 831 cases (2000-2007). Journal of the American Veterinary Medical Association, 241(12), 1617-1626. https://doi.org/10.2460/javma.241.12.1617

Aikawa, T., Fujita, H., Shibata, M., \& Takahashi, T. (2012). Recurrent thoracolumbar intervertebral disc extrusion after hemilaminectomy and concomitant prophylactic fenestration in 662 chondrodystrophic dogs. Veterinary Surgery, 41(3), 381-390. https://doi.org/10.1111/j.1532-950X.2012.00970.x.

Bergknut, N., Smolders, L. A., Grinwis, G. C. M., Hagman, R., Lagerstedt, A. S., Hazewinkel, H. A. W., Tryfonidou, M. A., \& Meij, B. P. (2013). Intervertebral disc degeneration in the dog. Part 1: Anatomy and physiology of the intervertebral disc and characteristics of intervertebral disc degeneration. The Veterinary Journal, 195(3), 282-291. https://doi.org/10.1016/j.tvj1.2012.10.024.

Brisson, B. A. (2010). Intervertebral disc disease in dogs. The Veterinary Clinics of North America. Small Animal Practice, 40(5), 829-858.

Castel, A., Olby, N. J., Mariani, C. L., Muñana, K. R., \& Early, P. J. (2017). Clinical characteristics of dogs with progressive myelomalacia following acute intervertebral disc extrusion. Journal of Veterinary Internal Medicine, 31(6), 1782-1789. https://doi.org/10.1111/jvim.14829.

Costa, R. C., De Decker, S., Lewis, M. J., Volk, H., \& Consortium, C. (2020). Diagnostic imaging in intervertebral disc disease. Frontiers in Veterinary Science, 7, 1-100.

Dewey, C. W., \& Costa, R. C. (2016). Practical guide to canine and feline neurology (3 Ed.). Wiley Blackwell.

Ettinger, S. J., Fedlman, E. C., \& Taibo, R. A. (2002). Tratado de medicina interna veterinaria: enfermedades del perro y el gato. Manole.

Falzone, C. (2017). Canine acute cervical myelopathy: Hydrated nucleus pulposus extrusion or intraspinal discal cysts? Veterinary Surgery, 46(3), 376-380. https://doi.org/10.1111/vsu.12631.

Fenn, J., Olby, N. J., \& Consortium, C. S. C. I. (2020). Classification of intervertebral disc disease. Frontiers in Veterinary Science, 7. https://doi.org/10.3389/fvets.2020.579025.

Hankin, E. J., Jerram, R. M., Walker, A. M., King, M. D., \& Warman, C. G. A. (2012). Transarticular facet 
screw stabilization and dorsal laminectomy in 26 dogs with degenerative lumbosacral stenosis with instability. Veterinary Surgery, 41(5), 611-619. https://doi.org/10.1111/j.1532-950X.2012.01002.x.

Hansen, H.-J. (1951). A pathologic-anatomical interpretation of disc degeneration in dogs. Acta Orthopaedica Scandinavica, 20(4), 280-293. https://doi.org/10.3109/17453675108991175.

Hansen, H.-J. (1952). A pathologic-anatomical study on disc degeneration in dog: With special reference to the so-called enchondrosis intervertebralis. Acta Orthopaedica Scandinavica, 23(Sup 11), 1-130.

Innes, J., \& Melrose, J. (2015). Embryology, innervation, morphology, structure, and function of the canine intervertebral disc. In J. M. Fingeroth \& W. Thomas (Eds.), Advances in intervertebral disc disease in dogs and cats. Wiley Blackwell.

Jeffery, N D, Levine, J. M., Olby, N. J., \& Stein, V. M. (2013). Intervertebral disk degeneration in dogs: consequences, diagnosis, treatment, and future directions. Journal of Veterinary Internal Medicine, 27(6), 1318-1333. https://doi.org/10.1111/jvim.12183.

Jeffery, N. D., Barker, A. K., Hu, H. Z., Alcott, C. J., Kraus, K. H., Scanlin, E. M., Granger, N., \& Levine, J. M. (2016). Factors associated with recovery from paraplegia in dogs with loss of pain perception in the pelvic limbs following intervertebral disk herniation. Journal of the American Veterinary Medical Association, 248(4), 386-394. https://doi.org/10.2460/javma.248.4.386.

Martin, S., Liebel, F. X., Fadda, A., Lazzerini, K., \& Harcourt-Brown, T. (2020). Same-day surgery may reduce the risk of losing pain perception in dogs with thoracolumbar disc extrusion. Journal of Small Animal Practice, 61(7), 442-448. https://doi.org/10.1111/jsap.13224.

Moore, S. A., Tipold, A., Olby, N., Stein, V. M., \& Granger, N. (2020). Current approaches to the management of acute thoracolumbar disc extrusion in dogs. Frontiers in Veterinary Science, 7, 1-15. https://doi.org/10.3389/fvets.2020.00610.

Olby, N. J., MacKillop, E., Cerda-Gonzalez, S., Moore, S., Muñana, K. R., Grafinger, M., Osborne, J. A., \& Vaden, S. L. (2010). Prevalence of urinary tract infection in dogs after surgery for thoracolumbar intervertebral disc extrusion. Journal of Veterinary Internal Medicine, 24(5), 1106-1111. https://doi.org/10.1111/j.1939-1676.2010.0567.x.

Risio, L. (2015). A review of fibrocartilaginous embolic myelopathy and different types of peracute noncompressive intervertebral disk extrusions in dogs and cats. Frontiers in Veterinary Science, 2(24), 1-9. https://doi.org/10.3389/fvets.2015.00024.

Risio, L., Adams, V., Dennis, R., \& McConnell, F. J. (2009). Association of clinical and magnetic resonance imaging findings with outcome in dogs with presumptive acute noncompressive nucleus pulposus extrusion: 42 cases (2000-2007). Journal of the American Veterinary Medical Association, 234(4), 495504. https://doi.org/10.2460/javma.234.4.495.

Salger, F., Ziegler, L., Böttcher, I. C., Oechtering, G., Böttcher, P., \& Flegel, T. (2014). Neurologic outcome after thoracolumbar partial lateral corpectomy for intervertebral disc disease in $72 \mathrm{dogs}$. Veterinary Surgery, 43(5), 581-588. https://doi.org/10.1111/j.1532-950X.2014.12157.x.

Sharp, N. J., \& Wheeler, S. J. (2005). Small animal spinal disorders: diagnosis and surgery. Elsevier Mosby.

Taylor, S. M. (2015). Distúrbios da Medula Espinhal. In R. W. Nelson \& C. G. Couto (Eds.), Medicina Interna de Pequenos Animais (pp. 1048-1072). Elsevier Brasil.

Histórico do artigo:

Recebido: 17 de outubro de 2020. Aprovado: 5 de novembro de 2020.

Disponível online: 18 de janeiro de 2021.
Licenciamento: Este artigo é publicado na modalidade Acesso Aberto sob a licença Creative Commons Atribuição 4.0 (CC-BY 4.0), a qual permite uso irrestrito, distribuição, reprodução em qualquer meio, desde que o autor e a fonte sejam devidamente creditados. 